\title{
Musiques et identités sénégalaises
}

Music and Senegalese identities

Músicas e identidades senegaleses

\section{Moussa Sy}

\section{CpenEdition}

\section{Journals}

Édition électronique

URL : https://journals.openedition.org/ries/5972

DOI : 10.4000/ries.5972

ISSN : 2261-4265

\section{Éditeur}

France Education international

Édition imprimée

Date de publication : 1 septembre 2017

Pagination : 97-105

ISBN : 978-2-85420-615-9

ISSN : $1254-4590$

\section{Référence électronique}

Moussa Sy, "Musiques et identités sénégalaises », Revue internationale d'éducation de Sèvres [En ligne], 75 | septembre 2017, mis en ligne le 01 septembre 2019, consulté le 24 juin 2021. URL : http:// journals.openedition.org/ries/5972 ; DOI : https://doi.org/10.4000/ries.5972 


\title{
Musiques et identités sénégalaises
}

\author{
Moussa Sy \\ Professeur d'éducation musicale, Sénégal
}

Il ne fait pas le moindre doute qu'il n'existe sur la terre aucun peuple plus naturellement sensible au son de la musique que celui-ci (peuple de la Sénégambie); les principaux personnages (c'est-à-dire les rois et les chefs) la considèrent vraiment comme un ornement de leur État, si bien que la musique fait rarement défaut lorsque nous leur rendons visite.

Richard Jobson,

The discovery of River Gambra (1623)

\section{Place et Rôle \\ DE LA PRATIQUE MUSICALE \\ DANS LA SOCIÉTÉ TRADITIONNELLE SÉNÉGALAISE}

En Afrique, la pratique musicale accompagne depuis toujours toutes les activités humaines. Le chroniqueur sénégalais Yoro Diaw (1847-1919), dans Les Cahiers de Yoro Diaw, signale que les rois et les chefs de provinces avaient des fara, dont le rôle était de jouer de la musique. Certains instruments servaient d'insignes aux autorités dans l'ancien empire du Djoloff, comme les dioungdioung (grands tambours bifaces), qui étaient fabriqués pour les empereurs et vice-rois des différentes provinces.

La musique traditionnelle animait les travaux des champs et plongeait le peuple dans les rêveries avec les khalam (guitare de quatre à cinq cordes), riiti (violon monocorde des bergers) ou autres thokoro (flûte peul), en rappelant l'histoire des fils des contrées et terroirs. Dans certains villages, la musique marquait les cérémonies des rites de passage, dans d'autres, une fête cyclique, etc. La musique traditionnelle africaine présidait aux différents événements importants de la vie : naissance, mariage, funérailles, événements religieux. Le répertoire se composait de chants d'amour, de chants satiriques, humoristiques, d'épopées relatant les exploits de héros et parfois de chants érotiques. Elle berçait les bébés et animait les jeux des enfants. La musique, essentiellement fonctionnelle, était à la base de tous les aspects de la vie.

La musique traditionnelle africaine se transmet oralement. La musique fait corps avec la tradition, qu'elle institutionnalise et formalise, voire rigidifie. L'instrument et l'artiste forment une unité, dans laquelle l'instrument devient 
la voix de l'artiste. Certains récits ne peuvent être transmis que par le chant. Comme il était d'usage dans la tradition, le jeune musicien se formait chez un maître. Cette formation obéissait à des règles strictement parentales ou sentimentales. La relation entre l'élève et le maître était de nature filiale, le plus souvent, ou de maître à disciple (parfois l'apprenant était obligé de développer ses connaissances au sujet d'une technique particulière ou d'une branche d'une généalogie). Le maître portait en lui son expérience comme un bien inaltérable et l'élève, une fois jugé apte à recevoir les cours, se devait d'être à la disposition de son professeur, jour et nuit. Pour acquérir le savoir du maître, il était, par la force des choses, obligé d'habiter avec lui pendant des années, son instrument toujours à portée de main. L'apprentissage devait englober tous les aspects non seulement du savoir strictement musical mais aussi - et de façon non moins importante - le volet humain dans tout son acception. En effet, le maître occupait une place particulière dans la chaîne qui relie l'homme à la surnature et au ciel ; il était celui qui peut rendre compte des musiques depuis le lieu où elles existent, celui des savoirs réservés, des savoirs acquis souvent au prix de l'initiation, avec le piège du secret dont celle-ci se pare et qui empêche l'initié de transmettre ce qu'il a vu ou ce qu'on lui a dit. L'élève acceptait de servir le maître et de l'aider dans les travaux champêtres ou autres, l'accompagnait dans ses voyages de pays en pays, chez les rois et princes, le secondait dans les cérémonies. L'apprentissage et l'éducation, toujours ensemble, se pratiquaient sans interruption, sans horaires fixes. Ce type de relation ne visait ni l'innovation ni l'originalité. Chaque communauté avait ses critères propres en matière d'éducation. Cette forme d'éducation, propre à la tradition orale, remplissait les fonctions attendues d'elle dans les sociétés africaines, où prédominent le rythme et la parole, la chaleur du mouvement vital par rapport à ce qui est fixé. Le rôle de ces "écoles " se limitait essentiellement à la reproduction "d'un esprit musical », il fallait n'être que la voix du maître, comme l'évoque Djibril Tamsir Niane (1960) :

Ma parole est pure et dépouillée de tout mensonge ; c'est la parole de mon père ; c'est la parole du père de mon père. Je vous dirai la parole de mon père telle que je l'ai reçue.

Ainsi, dans les traditions africaines, à côté du raisonnement et de la réflexion intellectuelle, la survivance du sentiment de l'honneur, de la tendance à l'esprit de solidarité ou de communauté, de religiosité, ont toujours été développés dans les apprentissages de la science musicale. La musique, de même que l'organisation sociale ou la langue ou encore la religion, représentait une des bases importantes de la société traditionnelle.

De nombreux témoignages racontés le soir au coin du feu permettent de mesurer toute la passion qui a animé ces musiciens durant des siècles. Les restitutions de mélodies témoignaient souvent d'une rare intensité sonore et sentimentale. Les airs s'apprenaient de façon routinière. C’est la répétition globale 
des mélodies, en vue de l'obtention d'un son désiré, qui constituait l'essentiel de l'apprentissage. Ceci explique comment certaines mélodies traditionnelles de structure complexe ont été assimilées intégralement d'une manière orale par beaucoup de musiciens d'autrefois. Ici intervient la motivation due au plaisir engendré par la musique.

\section{Le griot, médiateur entre les hommes et les forces de la nature}

En Afrique, la musique joue un rôle de médiation entre les hommes et les forces de la nature. Le griot était vu comme un personnage disposant de pouvoirs mystiques. Ainsi, chez les Wolof, la musique détient le pouvoir d'attirer les premières pluies avec le bawnaan ${ }^{1}$; de conjurer le mauvais sort avec le ndeup ${ }^{2}$, chez les Lébous; d'introniser un roi, de marquer le passage des jeunes garçons à l'âge adulte avec le boukout ${ }^{3}$ chez les Diola, etc. Les apprenants en musique traditionnelle devaient maîtriser la généalogie des familles et les liens familiaux, être à égale distance des composantes de leur communauté, savoir distinguer les musiques pour les nobles et les princes, celles des rites, pratiquer les instruments liés aux événements religieux ou profanes, sans oublier les connaissances ésotériques. Les instruments permettaient de communiquer avec les forces invisibles. Selon qu'ils étaient à cordes, à vent, à percussion, ils étaient en rapport avec les éléments : la terre, l'air et l'eau.

Rien ne se faisait autrefois sans un chant ou la présence du griot : Niani, chant de bravoure ; Tara, musique dédiée au marabout El hadj Omar FoutiyouTall ; Kéléfaba, guerrier mandingue ; ceddo, chant de révolte, etc. Ces classiques de la musique traditionnelle, où sont retracées les pages glorieuses de notre histoire et évoqués les exploits des grandes personnalités, faisaient rêver les jeunes, qui découvraient des personnages dont s'enorgueillissaient non seulement leurs familles mais toute leur communauté.

Le griot traditionnel prenait en charge la mélancolie et autres soucis de la destinée humaine en les transformant en chants. En un instant, il savait faire jaillir comme une étincelle un mot, de ce mot émergeait une note, et de cette note un chant. Le griot était celui qui savait regarder, observer les signes : la pluie, le retour de la verdure, ainsi que l'issue heureuse d'une bataille, pour les élever aux dimensions d'une fresque épique. Il n'excluait pas la souffrance, mais la transformait en conquête. Comme le dit Nimrod (2003), citant Kourouma, le griot était musicien, "savant, historien, généalogiste et panégyriste ».

\footnotetext{
1. Cérémonie incantatoire pour obtenir la pluie. (NdIR)

2. Pratique traditionnelle destinée à soigner les personnes perturbées psychiquement ou mentalement. (NdIR)

3. Rite d'initiation. (NdIR)
} 


\section{LA MUSIQUe AU SÉnÉGAL APRÈS L'INDÉPENDANCE}

Dans l'après-guerre, toutes les grandes villes sénégalaises avaient un ou plusieurs orchestres modernes. Les soirées étaient très animées. Les amoureux de la musique avaient élaboré de nombreuses stratégies pour développer leur art : incorporer l'armée, prendre des cours d'instruments chez des aînés jusque dans les bars, chez les missionnaires, fabriquer leurs propres instruments, entre autres. Dans les grandes villes, ils profitaient de la présence des étrangers (appelés effectuant leur service militaire ou fonctionnaires affectés par la métropole) et se formaient sur le tas. Souvent, les formations musicales étaient créées par les autorités municipales. Durant cette période post-indépendance, les vedettes de la musique traditionnelle étaient très sollicitées elles aussi, par les jeunes comme par leurs parents, pour des taneber ou khawaré (soirées de musiques traditionnelles avec chants et danses) et des panals (parades) ou autres événements. On jouait toutes sortes de musiques et même de la musique classique.

L'engouement pour la musique poussa les musiciens traditionnels vers les villes. Toutes les occasions étaient bonnes pour faire de la musique, des khawarés aux séances de lutte. Ce n'était plus seulement pour les événements traditionnels mais aussi pour les rencontres politiques. À chaque politicien était attaché un musicien.

Le musicien sénégalais n'éprouvait pas le besoin de prendre des cours académiques de musique; il se contentait de reprendre les œuvres étrangères. Et selon l'âge ou le niveau de ses connaissances, l'imitation aboutissait souvent à une création. Ainsi, plusieurs pièces de musique européenne sont devenues des morceaux à l'ambiance bien «sénégalaise ».

L'organisation des orchestres modernes et leur mode de gestion inspirait beaucoup les musiciens traditionnels. Les formations se créaient pour des raisons autres que familiales, ethniques, géographiques, etc. Le Président-poète Léopold Sédar Senghor, voulant capitaliser toute cette énergie, créa alors l'École des arts.

\section{L'ENSEIGNEMENT DE LA MUSIQUe À L’ÉCOLE}

L'enseignement musical est présent dans le programme sénégalais, de la maternelle au secondaire. À la maternelle ou plus exactement au préscolaire et dans le primaire depuis 1972, le but est « de favoriser la libre expression et le développement du goût de l'enfant dans les activités musicales ", tandis que dans le secondaire, il est recommandé « de chanter en chœur et de donner le goût de la musique ». Il s'y ajoute l'histoire de la musique, en plus des connaissances théoriques et pratiques.

L'éducation musicale était pratiquée au Sénégal, bien avant l'indépendance, particulièrement dans l'enseignement confessionnel, sous la forme de cours de chants, avec un répertoire français : Au clair de la lune, Sur le pont d'Avignon, Le Furet, par exemple. 
Avec la création du conservatoire de Dakar, un corps d'éducateurs artistiques est créé, qui sera suivi, plus tard, du corps des professeurs d'éducation musicale. Le programme reste européen mais le cours va avoir deux volets : la théorie et l'histoire.

Pourtant, l'éducation musicale occupe aujourd'hui une position que l'on peut qualifier de fragile, voire marginale, à en juger par certains aspects : budget alloué à la formation des enseignants au conservatoire, horaires attribués à cet enseignement dans les établissements scolaires, une discipline qui reste facultative, le manque de matériel, sans oublier des classes pléthoriques et le sentiment de malaise et d'abandon des enseignants d'éducation musicale.

\section{Langues et musiques du Sénégal}

On dénombre au Sénégal ${ }^{4}$ une vingtaine d'ethnies, dont quelques-unes ont été retenues comme principales par l'adoption de leur langue comme langues nationales : par exemple, les ethnies Wolof (Wolof, Lébou), Halpulaar (Fouta, Laobé, Peul et Toucouleur), Mandingue (Malinké, Socé), Sérère et Joola.

Les Wolofs ont donné au pays sa langue principale et des lettres de noblesse aux percussions sénégalaises ; ils sont le peuplement dominant et donnent une ambiance de fête à la musique sénégalaise, avec leurs sabars (tambours) aux rythmes très dansants. Le wolof doit d'être langue dominante à ses sonorités, qui indiquent très musicalement la joie ou l'inquiétude. La musique des Halpulaar, peuple du yéla ${ }^{5}$, rappelle le «blues »; les peuls se localisent dans le Fouta, sur les cours moyens du fleuve Sénégal et en Haute Casamance. Les Sérères, dont la musique semble être de la poésie, peuplent la région du Sine et du Saloum en particulier. Les Mandingues, communautés de djélys (griots) et de balla (joueurs de balafon), plus portés vers la musique de cour, avec des grands maîtres de la parole, dominent dans la moitié sud du Sénégal, en Moyenne Casamance. Le Sénégal compte aussi des minorités : les Soninkés, le groupe Tenda constitué de Badiananké, Koniagui, Bassari et de Bedik, les Baïnouk, les Joola, les Balantes.

\section{Finalités et pédagogie de l'enseignement de la musique au Sénégal}

Le curriculum en cours d'implantation au collège invite à l'adoption d'une pédagogie moderne, fondée sur l'approche par les compétences, de pratiques éducatives contextualisées mais aussi ouvertes à l'environnement local et extérieur. La pratique musicale peut et doit ainsi aider à la construction d'une culture et à la formation esthétique.

4. La population du Sénégal est estimée à 15 millions de personnes actuellement.

5. Le Yéla est la musique principale des Toucouleurs ; c'est un ensemble de chants et de danses qui, à l'origine, servait de rituel pour rendre grâce à Dieu. (Source : d'après Wikipédia, NdIR) 
La pratique musicale, dans le système éducatif scolaire actuel, prend en compte et développe la pluralité et la diversité des aptitudes, tout en favorisant la formation d'une société unie et attachée à sa culture. Dans le nouveau programme d'éducation musicale, il est d'ailleurs suggéré que l'enseignant prenne en compte les éléments culturels spécifiques de sa zone. Celui qui est en Casamance doit privilégier la musique de son aire géographique, tout en s'appuyant sur une approche comparative avec les autres aires culturelles. Les enseignements doivent montrer la particularité régionale mais aussi les points communs. Certaines activités portant sur la pratique musicale favorisent l'usage d'éléments extérieurs (par exemple, avec les instruments de musique, le mélange très intéressant des percussions Wolofs et Diolas et/ou les cordes peuls).

Les exercices et activités se font avec beaucoup de naturel chez les tout petits. L'activité vocale aide à la découverte, à l'apprentissage et à la mémorisation des phonèmes des signes linguistiques, voyelles, consonnes, mots, etc. Nous continuons l'apprentissage de chants européens (pour la diction et un prolongement de la lecture) et ceux d'autres continents, car ne voulons-nous pas être des citoyens du monde? Les chants en langue nationale ont eu un impact réel sur la scolarisation. L'école commence à devenir un prolongement de la maison. Pour la lecture et le langage, la musique améliore la perception au niveau des sonorités, des syllabes et aide ainsi aux travaux de phonétique et de diction. Nous essayons de lier les exercices rythmiques en percussion et les nombres de syllabes chantées, afin d'amener les tous petits à ne pas dépasser les frappes; les collégiens situent les temps plus facilement. Dans les contes africains, il existe toujours une partie chantée qui résume l'histoire et facilite la compréhension du texte. Cette activité est utilisée comme moment d'écoute. À travers les jeux, les enfants, très tôt et dans toutes les langues locales, s'amusent avec les chiffres et les nombres.

\section{MUSIQUE ET MUTATIONS DE LA SOCIÉTÉ SÉNÉGALAISE}

De nos jours, la culture ne sait plus très bien ce qu'elle représente ni à qui elle s'adresse. Les techniques traditionnelles des peuples ne conviennent plus à la "communication ». Comme le disait Ravi Shankar dans un entretien à Munich, en août 1972 : "le musicien doit être fidèle aux traditions du passé, sincère dans son attitude vis-à-vis du présent et capable d'animer son art de toutes les émotions afin qu'il puisse agir sur son auditeur»(Unesco, 1973).

Si nos valeurs ont pu résister et survivre, c'est grâce aux griots. Le griot, en effet, avait un rôle très important dans sa société. Il était le dépositaire de la tradition orale et historique. Car la pratique musicale est avant tout sociale, un mode de vie permettant aux acteurs d'en tirer des éléments régénérateurs. Si nous avons quelque chose qui nous est propre, nous le retrouvons certainement dans notre musique. 
Récemment encore, l'on estimait que la musique traditionnelle n'avait comme préoccupation que l'exaltation. La ferveur suscitée par l'épopée était une manière de tirer les choses vers le haut. Aujourd'hui, les questions de généalogie n'ont plus le même effet car le statut social du Guer (le noble) ne dépend plus de sa lignée mais de son compte en banque. Le griot n'est plus celui qui, en abordant la généalogie de telle ou telle lignée, fait revivre un passé. Il anime aujourd'hui pour tout le monde et subit les lois de la concurrence. Ainsi cette transformation sociale, cette "décorporalisation » ont mené vers l'autonomie de la nouvelle génération de jeunes griots d'abord, puis des griots en général, avec la création d'autres rapports dominés par l'argent. Il faut bien vivre.

Le degré d'évolution et le niveau de vie de chaque société influencent le développement de l'art musical et des instruments en particulier. Aujourd'hui, nous ne pouvons nier l'orientation artistique et culturelle que prend la société sénégalaise, entraînant irrémédiablement la musique dite traditionnelle dans son mouvement. L'ère du numérique semble avoir pris le dessus.

Beaucoup plus pragmatiques que leurs aînés, les jeunes, aujourd'hui, ont une conception très moderne de leur rapport à la culture et à la société. La radio a envahi tous les espaces, elle est la compagne des bergers, la confidente des agriculteurs; la télévision est partout. Internet est devenu familier. Les médias ont tout simplement remplacé les griots et rendent la musique accessible à tous. Et, ce qui est très important, la musique devient commercialisable et rentable.

\section{Un regain d'intérêt pour l'éducation musicale grâce aux nouvelles technologies}

Les enseignants de musique sont conscients d'avoir un rôle important à jouer dans la formation de ceux qui formeront demain la société sénégalaise.

Aujourd'hui, la recherche peut permettre à l'éducation musicale d'améliorer et de varier ses approches pédagogiques, voire de créer des méthodes inspirées de l'enseignement traditionnel. Selon des travaux récents, la musique renforcerait les capacités de transfert des connaissances. Elle agirait comme un catalyseur qui stimule le développement des capacités cognitives des enfants et intègrerait admirablement les divers aspects de la vie sociale et religieuse. La plupart des chansons, comptines ou fables peuvent être des guides pour la classe d'âge concernée. Les paroles sont des maximes ou des proverbes, des phrases avec des mots aux sonorités très musicales qui permettent d'apprendre à vivre en société, à vivre avec les autres.

Depuis 2013, avec l'apport de la technologie, il s'agit de redonner un nouvel élan à la pratique musicale dans les établissements scolaires, en permettent aux élèves d'apprendre la musique sénégalaise avec une approche moderne. 
De nouvelles musiques, un nouveau statut du musicien, un public plus large, l'éclosion des nouvelles technologies : il faut prendre la mesure de cette nouvelle situation. Les mobiles, tablettes et ordinateurs portables apparaissent dans le cours d'éducation musicale. Utiliser un instrument devient facile grâce aux logiciels et applications. Ainsi, les compétences sont diversifiées. Nous avons agréablement constaté un intérêt nouveau pour le cours de musique de toutes les catégories d'élèves. Certains se sentent obligés d'acheter des outils plus performants, d'autres commencent à changer d'attitude : ils prennent des notes avec leurs outils numériques, prennent en images certains aspects du cours au lieu d'écrire. De nouvelles compétences se révèlent. Des groupes se forment, de dimensions différentes, selon les travaux, les compétences, etc. Certaines familles contribuent à l'achat du matériel. Il semble que le travail soit plus régulier, continu. Les travaux à faire à la maison sont désormais les bienvenus. La tradition n'est pas oubliée. Ainsi, dans les clubs de musique, les cours de musique réhabilitent des chants comme les «kassaks» pour les garçons (la circoncision) et le «ndiam» ou tatouage chez les filles.

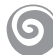

L'éducation musicale se donne comme objectif de cultiver à la fois un esprit, une sensibilité, un corps. L'importance d'un engagement aussi large que possible, englobant la sensorialité, l'affectivité, les capacités intellectuelles, la participation corporelle, mettant l'accent sur le rôle que la musique est susceptible de jouer dans le développement humain, doit être défendue et mise en valeur.

La pédagogie a une fonction sociale. Aucune action pédagogique ne peut être coupée ou isolée d'un environnement en perpétuel changement.

Dès la création de la Commission nationale de l'éducation musicale, nous avons plaidé pour que les enseignants de musique puissent s'initier à de nouvelles connaissances et techniques éducatives. Au Sénégal, hormis dans un ou deux établissements, les approches pédagogiques sont méconnues. Nous avons exprimé le vœu que l'École des arts, qui est la case formatrice des éducateurs musicaux, mette en place un laboratoire de recherche pour prendre en charge toutes les questions liées à la pédagogie de la musique. Car les méthodes d'enseignement et la démarche doivent changer, si l'on veut susciter chez les élèves des conduites novatrices. 


\section{BibliographiE}

BEART C. (1955) : "Jeux et jouets de l'Ouest Africain », Mémoires de l'Institut français d'Afrique Noire, tome 1, IFAN-Dakar, $\mathrm{n}^{\circ} 42$.

BENCHEICH A. (1975): Formations économiques et sociales et pratique musicale, Alger, éditions ENAP.

GOUVERNEMENT DU SÉNÉGAL (1972): décret n 72-861 du 13 juillet 1972 «Réforme de l'enseignement au Sénégal ».

GADEN H. (éd) (1912) : "Légendes et coutumes sénégalaises. Cahiers de Yoro Dyao ", Paris : E. Leroux, consultable en ligne (19 p.) : [https://goo.gl/BHXMkD]

GRABOCZ M. (sous la direction de) (1999) : Méthodes nouvelles, musique nouvelles: musicologie et création, Presses universitaires de Strasbourg.

NIMROD (2003) : «La servitude volontaire de l'écrivain africain », Africultures, 54, (1), 194-198, DOI: 10.3917/afcul.054.0194.

ROSSELOT B. (2001) : Aventuriers et Griots: De la galère à la profession, Paris : L'Harmattan.

SAMB A. (1975) : «Folklore wolof du Sénégal », Bulletin de l’IFAN, tome 37, série B, $\mathrm{n}^{\circ} 4$.

SANKHARE O. (1998) : Youssou Ndour le poète, Dakar : Nouvelles éditions africaines du Sénégal.

SOUTHERN E. (1997): The Music of Black Americans: A History, $3^{\mathrm{e}}$ édition, New York : Norton\& Company.

TAMSIR NIANE D. (1960): Soundjiata ou l'épopée mandingue, Paris: Présence africaine.

UNESCO (1973) : Revue Cultures, « Musique et société », vol. 1, nº 1, Unesco. 
